\title{
Behavior of Positrons
}

\section{in the HfNbTaTiZr Complex Concentrated Alloy}

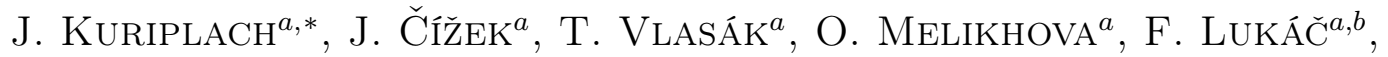 \\ J. ZÝKA ${ }^{c}$ AND J. MÁLEK ${ }^{c}$ \\ ${ }^{a}$ Department of Low Temperature Physics, Faculty of Mathematics and Physics, Charles University, \\ V Holešovičkách 2, CZ-18000 Prague 8, Czech Republic \\ ${ }^{b}$ Department of Materials Engineering, Institute of Plasma Physics of CAS, \\ Za Slovankou 1782, CZ-18200 Prague 8, Czech Republic \\ ${ }^{c}$ UJP PRAHA a.s., Nad Kamínkou 1345, CZ-15610 Prague 5 - Zbraslav, Czech Republic
}

\begin{abstract}
The properties of positrons delocalized in the HfNbTaTiZr complex concentrated alloy are investigated theoretically and related to available experimental data. Positron lifetimes calculated for random atomic arrangements appear to be short compared to the experiment carried out on a vacancy-free sample. Contrarily, a configuration exhibiting a local atomic order provides a lifetime that is very close to the experimental value. These observations indicate that the local atomic order is occurring in the studied alloy, in agreement with other measurements. The analysis of calculated results shows that the positron lifetime and affinity are affected to some extent by the alloy substitutional disorder, lattice relaxations, and charge transfer among atomic species, which should be taken into account in precise calculations.
\end{abstract}

DOI: 10.12693/APhysPolA.137.260

PACS/topics: 61.66.Dk, 78.70.Bj, 65.40.gd, 71.60.+z

\section{Introduction}

Complex concentrated alloys (CCAs) [1] attract wide attention at present. They are supposed to replace, or at least supplement, the current portfolio of alloys based mostly on one element (or at most two elements) with small additions of other atomic species. A simple example can be a steel that is based on iron to which other elements are added to serve a specific steel purpose. On the other hand, CCAs are based on several (or many) elements with a substantial concentration, which could bring novel and improved alloy characteristics. CCAs are also known as high entropy alloys (HEAs) [2] since ideally random alloys with several constituting elements exhibit high configurational entropy, which was supposed to stabilize a simple, high temperature phase even at lower temperatures. At the time of HEA concept development, it was not obvious to which extent the tendencies to short range order - which reduces the configurational entropy - might be important. The enthalpy of mixing various elements also plays a role in thermodynamic considerations for CCAs/HEAs.

The HfNbTaTiZr alloy was introduced by Senkov et al. [3]. The alloy is composed of refractory metals in equiatomic concentrations and its expected melting temperature is about $2500 \mathrm{~K}$. The envisaged use of the alloy possessing the bcc structure was originally as a material for aerospace industry (high temperature applications) and possibly also as a biocompatible material.

*corresponding author; e-mail: jan.kuriplach@mff.cuni.cz
Mechanical properties of the alloy at room temperature appear to be promising, which is not exactly true at elevated temperatures (see [4] for details). Since understanding the microstructure of the alloy is of fundamental importance to comprehend its other properties, we perform selected structure simulations and evaluate how the "alloying effects" influence the positron behavior.

Thus, in this paper, we investigate theoretically and computationally basic positron characteristics of the HfNbTaTiZr alloy. It is not obvious how positrons interact with a substitutionally disordered system where lattice relaxations play an important role. One can anticipate that the positron distribution is not perfectly periodic even if atoms constitute nominally a bcc lattice (as seen by the X-ray diffraction (XRD) [3, 5]). An important aspect in such considerations is the coherence length [6] of the positron wave function/packet. This length describes the linear size of a region where the wave function/packet remains coherent during the time evolution of the system, i.e., between the two subsequent (free) jumps of the positron in thermal equilibrium. The coherence length is thus related to the mean free path of positrons $\left(l_{+}\right)[7]$ in the alloy. In general, $l_{+}$'s in (vacancy-free) alloys do not apparently differ from those in pure metals or intermetallic compounds, as can be deduced from the measurements of the positron diffusion length (see, e.g. [8-10]). Thus, the order of magnitude of $l_{+}$in alloys is $100 \AA$ (at room temperature) and so is the coherence length. This justifies using the supercell approach in the computational positron investigations since the supercell size (typically 10-20 $\AA$ ) is smaller than the positron coherence length. In the case of positrons trapped at vacancies, the positron wave function 
is localized in the vacancy and its close vicinity, and using supercells is also well warranted (positrons do not jump). If the positron coherence length in metals was smaller than discussed here, then an approach based on scattering theory should be more effective to determine positron properties.

Other aspects to consider in a theoretical study of the positron behavior in alloys are as follows. The positron density is supposed to be enhanced in regions where lattice relaxations result in the formation of a larger (than average) interstitial space. The effect of charge transfer among atoms of different types does not need to be negligible. During its lifetime, the positron stays temporarily in different atomic environments - due to alloying and positron jumps - and an average of positron characteristics over several atomic configurations (supercells) should be performed. Such effects likely influence the positron lifetime, affinity, and momentum distribution of electron-positron pairs.

\section{Methods}

The random atomic configurations of the HfNbTaTiZr alloy with (nearly) equiatomic concentrations of constituting elements were prepared considering 128 atom supercells (i.e. $4 \times 4 \times 4$ bcc cells). In total, 13 different configurations (named 01, 02, ., 13 in the following) were constructed by putting atoms randomly at the ideal bcc positions. The concentrations of $\mathrm{Hf}, \mathrm{Nb}, \mathrm{Ta}$, $\mathrm{Ti}$, and $\mathrm{Zr}$ elements were kept in the 19.5 to 20.3 at.\% range (25 or 26 atoms of each type in the supercell), and all possibilities for the composition were taken into account. Obtained supercells were relaxed by means of Vienna ab initio simulation package (VASP) [11, 12] considering the so-called PBEsol [13] exchange-correlation functional. This means that atomic positions were optimized with respect to the total energy, keeping the supercell volume and shape constant. The bcc lattice constant $a=3.4089 \AA$ of the HfNbTaTiZr alloy was taken from an earlier measurement [5]. The atom/ion charge analysis after Bader [14] allows assigning charges to atoms in a physically plausible way using the concept of "zero flux surface" of the electron density around atoms. Such an analysis following the approach from [15] for VASP was performed for selected atomic configurations.

The effect of a local (short range) atomic order (LO) was also examined. We employed a Monte Carlo (MC) approach for this purpose, taking a configuration based on configuration 01 (see below). The simulation is carried out in the way that a new configuration with two randomly chosen atoms (of different types) exchanged is produced, re-relaxed, and "accepted" for the subsequent simulation step if its total energy is lower than that of the original configuration. If the energy is higher, some other random atomic exchange is attempted. Many hundreds of such MC iterations are necessary to become close to equilibrium. Such an equilibrium can be recognized by the observation that random exchanges are rarely accepted. Effectively, our MC algorithm is a variant of the
Metropolis Monte Carlo approach [16] at zero temperature. For the present study, we used a $300 \mathrm{~K}$ Metropolis MC configuration (derived from the configuration 01) described in [17] as an actual starting point. About 600 $\mathrm{MC}$ iterations (at $0 \mathrm{~K}$ ) were then performed. The configuration obtained in this way will be called "LO MC configuration" in the following.

Positron calculations were performed following the scheme described in $[18,19]$. In particular, VASP relaxed alloy configurations were processed using the WIEN2k code $[20]$ in order to obtain the precise electron densities and the Coulomb potentials to be used in the solution of the positron Schrödinger equation. The electronpositron correlation potential and enhancement factor were treated according to [21] where a precise, parameterfree gradient correction scheme for positrons was suggested. Here, we examine the positron lifetime and affinity and restrict ourselves to delocalized positron states. The positron lifetime brings information about the electron density and its variation inside supercells employed to simulate the HfNbTaTiZr alloy. The positron affinity is a sensitive tool to detect $[19,22]$ the local order in the studied alloy. The estimated numerical precision of calculations is $0.2 \mathrm{ps}$ for lifetimes and $0.01 \mathrm{eV}$ for affinities (cf. [18, 19]).

\section{Results and discussion}

Table I collects results of previous calculations presented in [19] where the positron lifetimes and affinities for the alloy constituting elements were determined considering their room temperature experimental structures and lattice parameters. Corresponding atomic metallic radii $\left(r_{\text {met }}\right)$ are also given (derived from the structures). First, we can observe that metallic radii are quite similar for all elements though $\mathrm{Zr}$ and $\mathrm{Hf}$ have largest values, and $\mathrm{Nb}$ and Ta possess the smallest ones. The deviation between the largest and smallest $r_{\text {met }}$ 's is $11 \%$ of the average, which translates to a $25 \%$ (27\%) difference between the largest and smallest positron lifetime (affinity). The larger is $r_{\text {met }}$, the longer (smaller) is the lifetime (affinity). This is a general trend, though there can be slight deviations due to the crystal structure and properties of individual atoms. The alloy metallic radii is just $0.02 \AA$ smaller than the average. These considerations indicate that the HfNbTaTiZr alloy lifetime should be slightly shorter (by about $4 \mathrm{ps}$ ) than the average $138.3 \mathrm{ps}$. Analogously, the positron affinity of the alloy is expected to be around $-3.83 \mathrm{eV}$. The agreement of calculated lifetimes presented in Table I with known experimental data is fair (except Hf for which no experiment has likely been done yet) and will be discussed in a future publication.

The positron lifetime results for 13 random relaxed configurations with 128 atoms are summarized in Fig. 1. The configurations studied are numbered from 01 to 13 , as indicated in the figure. The positron lifetimes vary in the range from 141.3 to 143.2 ps and the corresponding lifetime distribution is given in the inset of Fig. 1, 


\section{TABLE I}

Calculated positron lifetimes and affinities for constituting elements (after [18]), their crystal structures and structure-derived metallic radii. The row marked as 'average' shows average values (when applicable). Likewise the last row 'alloy' gives the structure and corresponding average metallic radius (lifetime and affinity depend on the atomic arrangement; see the text)

\begin{tabular}{l|c|c|c|c}
\hline \hline Element & Structure & $r_{\text {met }}[\AA]$ & $\tau[\mathrm{ps}]$ & $A_{+}[\mathrm{eV}]$ \\
\hline $\mathrm{Hf}$ & hcp & 1.58 & 147.5 & -4.07 \\
$\mathrm{Nb}$ & $\mathrm{bcc}$ & 1.43 & 123.0 & -3.57 \\
$\mathrm{Ta}$ & $\mathrm{bcc}$ & 1.43 & 117.4 & -3.34 \\
$\mathrm{Ti}$ & $\mathrm{hcp}$ & 1.46 & 145.8 & -4.36 \\
$\mathrm{Zr}$ & $\mathrm{hcp}$ & 1.60 & 157.6 & -4.40 \\
average & - & 1.50 & 138.3 & -3.95 \\
alloy & $\mathrm{bcc}$ & 1.48 & - & -
\end{tabular}

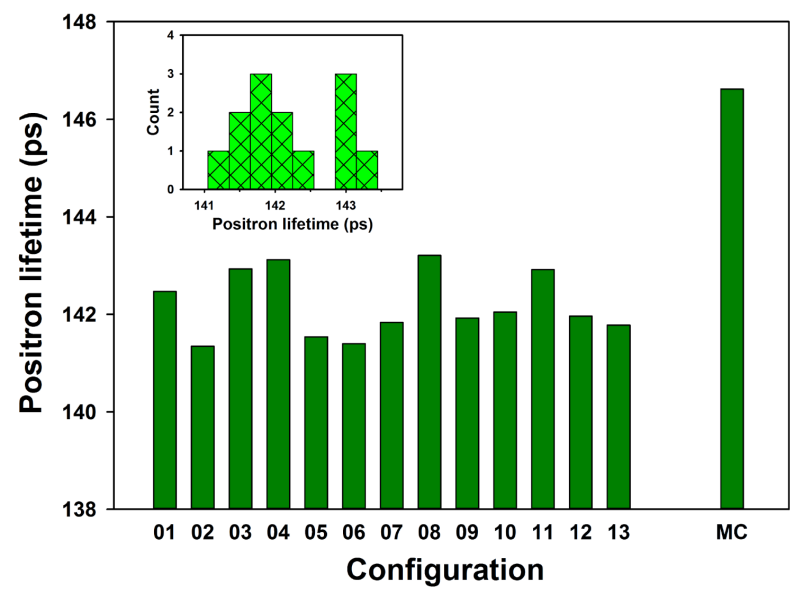

Fig. 1. Calculated positron lifetimes for random configurations 01 to 13 and the LO MC configuration. The inset shows the distribution of positron lifetimes for random configurations.

exhibiting a somewhat bimodal character. The average positron lifetime is $142.2 \mathrm{ps}$ and the distribution width is about $1.5 \mathrm{ps}$ (full width at half maximum, FWHM). While comparing this average to the experimental value $(146 \pm 1)$ ps obtained from the analysis of a one component spectrum [23], we can state a relatively good agreement of experiment and calculations, but the experiment is slightly shifted to longer lifetimes. Assuming that there were no defects capable to trap positrons which should be yet verified - we can anticipate that the effect is due to a local order present in the sample studied. Indeed, inspecting the LO MC configuration lifetime value shown also in Fig. 1 (146.6 ps) clearly points to a positron affinity effect when positrons prefer some regions of the sample/configuration. In this case it is in the $\mathrm{Zr}+$ Hf-rich regions, as discussed in more detail below. One can also see that the estimate of the random alloy bulk lifetime given above (related to Table I) is imprecise due to the charge transfer and lattice relaxation effects, also examined below.

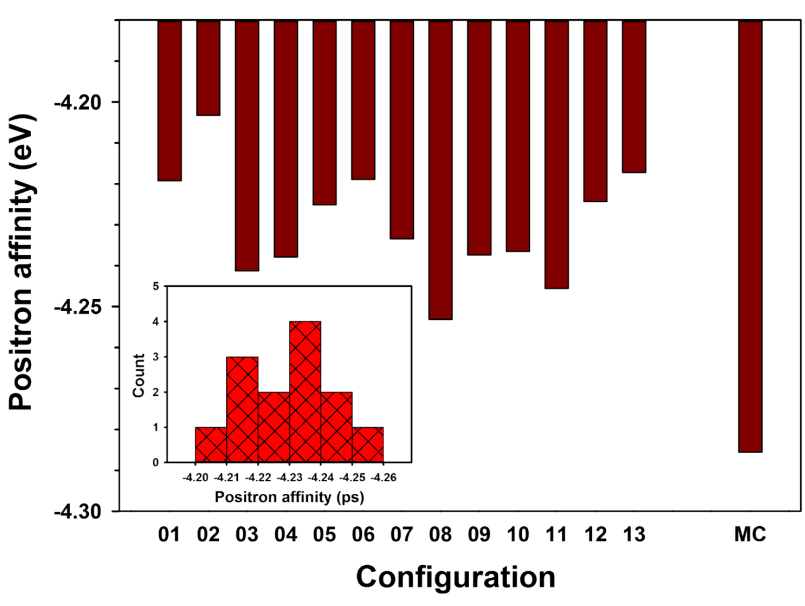

Fig. 2. Calculated positron affinities for random configurations 01 to 13 and the LO MC configuration. The inset shows the distribution of positron affinities for random configurations.

Concerning the positron affinity, Fig. 2 presents the results of $A_{+}$calculations for random alloy configurations (01-13) and the LO MC configuration. In the case of random configurations, $A_{+}$varies in the range from -4.25 to $-4.20 \mathrm{eV}$, with a distribution width (FWHM) of about $0.04 \mathrm{eV}$. The distribution is shown in the inset of Fig. 2. The bimodal character of the distribution can be again observed (cf. Fig. 1). The average value of the positron affinity amounts to $-4.23 \mathrm{eV}$ and is well separated from the value $-4.29 \mathrm{eV}$ obtained for the LO MC configuration. This again points to a preferred positron occupation of $\mathrm{Zr}+\mathrm{Hf}$-rich regions since the affinity of the LO MC configuration is closer to that of hcp constituting elements (see Table I) than bcc elements (the role of $\mathrm{Ti}$ is discussed below). Again, as in the case of lifetime, the estimate of $A_{+}$given above, based on elemental values, is not accurate, indicating non-negligible effects of alloying on the positron behavior. It should be mentioned that there is a general correlation between the lifetime and affinity for random configurations and LO MC one (not shown here): when the lifetime increases, $A_{+}$decreases, as expected. Nevertheless, there is a scatter in the lifetime vs. affinity dependence.

Figure 3 shows random configuration 01 together with the positron density plotted in one of (001) atomic planes. One can observe somewhat irregular character of the positron density, which is due to the substitutional disorder and relatively large atomic relaxations. The local minima of the positron potential correspond to local maxima of the density which occur in the interstitial space between atoms. Closer inspection reveals that the global maximum of the positron density correspond to interstitial sites surrounded mostly by Ti and other "hcp atoms" (more configurations were examined in this respect). The calculated positron lifetime and affinity are 142.5 ps and $-4.22 \mathrm{eV}$, respectively. 


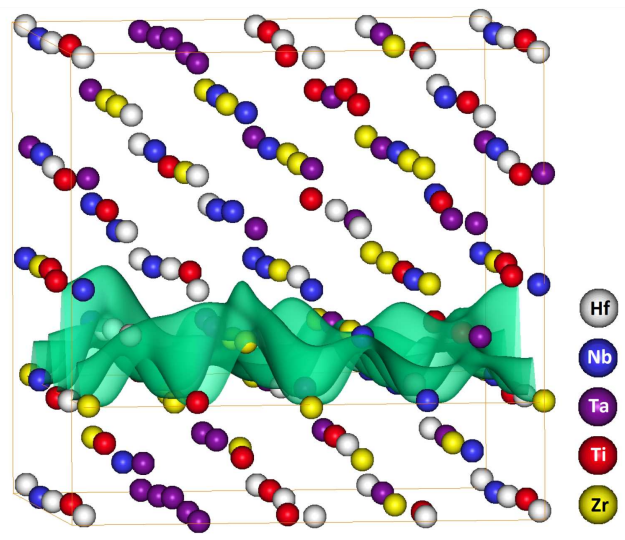

Fig. 3. Relaxed random alloy configuration 01 with the positron density height plot (green surface).

Another aspect of the alloy and positron (charge) distribution is the charge transfer among atomic species involved, which appears to be quite large. Indeed, the charge analysis after Bader [14] shows that for the relaxed configuration 01 , the average charges are $+0.28 e$, $-0.45 e,-0.75 e,+0.47 e$, and $+0.47 e$ for $\mathrm{Hf}, \mathrm{Nb}$, Ta, $\mathrm{Ti}$, and $\mathrm{Zr}$, respectively (with $e$ being the elementary charge). This means that atoms with nominally three $d$-electrons are getting electrons from those with nominally two $d$-electrons, which is not exactly the expected behavior. The point could be - as suggested by the "one atom" model $[24,25]$ — that electrons from some states of "hcp atoms" are transferred to "bcc atoms" to lower the energy of the system. In other words, the "hcp atoms" do not "like" to stay in the cubic environment.

The effect of relaxations on the positron characteristics examined is apparent. Figure 4 visualizes nonrelaxed configuration 01; i.e., atoms were not allowed to move from the ideal bcc lattice positions. The positron density behaves in a different way in the same plane compared to the relaxed configuration (cf. Fig. 3), and the lifetime and affinity are now $139.9 \mathrm{ps}$ and $-4.28 \mathrm{eV}$. The positron lifetime is shorter (by $2.6 \mathrm{ps)} \mathrm{with} \mathrm{respect} \mathrm{to}$ the relaxed case, as expected. This shows the effect of atomic relaxations in the alloy, which prolong the lifetime because of formation of larger interstitial spaces at some places. On the other hand, the affinity value does not fit in this trend, which is likely due to the fact that the configuration under study is not in equilibrium (i.e., is not relaxed with respect to atomic positions). The point is that the positron affinity depends on the Fermi energy (electron chemical potential), which depends on the atomic positions and thereby on forces acting on atoms which are nonzero when the configuration is not relaxed. This likely causes the observed effect which should be further investigated in the future.

In the case of the configuration exhibiting local order obtained using the Monte Carlo simulations (LO MC), one can clearly see regions which are $\mathrm{Nb}+\mathrm{Ta}$-rich and $\mathrm{Hf}+\mathrm{Zr}$-rich, as demonstrated in Fig. 5. Ti atoms appear to be rather located at interfaces between these

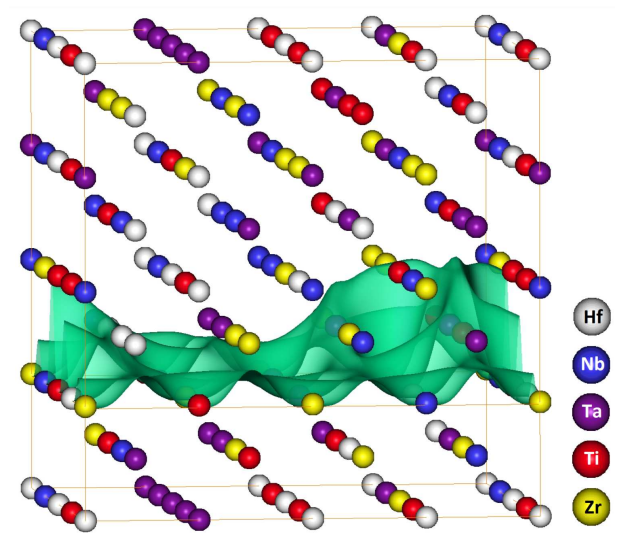

Fig. 4. Nonrelaxed random alloy configuration 01 with the positron density height plot (at the same position as for the relaxed configuration).

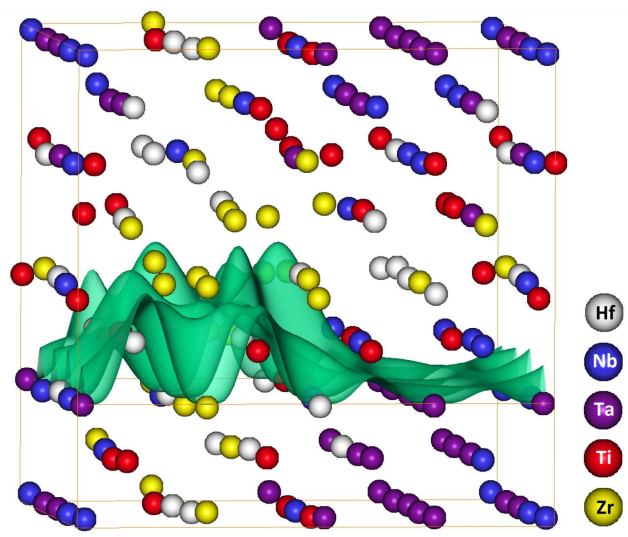

Fig. 5. The LO MC configuration with local order. The positron density in enhanced in the $\mathrm{Hf}+\mathrm{Zr}$-rich regions.

two regions. Moreover, the figure seems to indicate orientational relationship between the $\mathrm{Nb}+\mathrm{Ta}$-rich and $\mathrm{Hf}+\mathrm{Zr}$-rich regions (see the lower right corner). Namely, the $\{110\}$ atomic planes of the $\mathrm{Nb}+\mathrm{Ta}$-rich region (bcc phase) are parallel with the $\{110\}$ planes of the $\mathrm{Hf}+\mathrm{Zr}$-rich region, which would be likely transformed into an hcp phase if simulation conditions allow that. This is compatible with findings by Chen et al. [26], where the phase separation of the HfNbTaTiZr alloy into a bcc $(\mathrm{Nb}+\mathrm{Ta}-\mathrm{rich})$ and an hcp $(\mathrm{Hf}+\mathrm{Zr}$-rich $)$ phase has been observed and analyzed. The positron density plot (see the same figure) unveils that the positron density is enhanced in the regions where $\mathrm{Zr}$ and $\mathrm{Hf}$ dominate and is diminished in the parts with an increased concentration of $\mathrm{Nb}$ and $\mathrm{Ta}$. These effects explain why the positron lifetime and affinity are shifted towards corresponding values for $\mathrm{Hf}$ and $\mathrm{Zr}$ compared to random configurations. The average atomic charges of atomic species for the LO MC configuration are about the same as for the configuration 01 (see above) though their magnitudes are somewhat lower (except $\mathrm{Nb}$ ). This shows that LO does not significantly affect charge transfer effects. 
Finally, we make a methodological comment concerning the positron lifetime calculations using the atomic superposition/superimposed method (ASM) [27]. This method is obviously not capable to account for charge transfer effects (especially if they are large). Even if it was not investigated in detail, the ASM yields lifetimes which are 5-6 ps longer compared to fully selfconsistent calculations. This is too much for precise lifetime assessment, which renders the ASM approximate and usable for estimates only, at least in the present case.

\section{Conclusions}

The computational study carried out shows that the experimentally observed positron lifetime in the bulk HfNbTaTiZr alloy obtained by arc melting and subsequent etching does not contain open volume defects, but due to a local order positrons prefer regions with enhanced $\mathrm{Zr}$ and $\mathrm{Hf}$ content, which results in a slightly longer lifetime compared to the true random alloy. The positron affinity exhibits similar effects and should, in principle, be able to confirm such a local order, if measured accurately. The detailed examination of the positron density in random and locally ordered atomic configurations shows that the positron distribution (like other positron characteristics) are affected by the alloy disorder, lattice relaxations and charge transfer among atomic species. Since charge transfer effects are not negligible, the atomic superposition method provides just an estimate of the positron lifetime.

The experimental momentum distribution of electronpositron pairs also seems to reflect the local order, but the results have to be yet examined in detail and will be presented in a follow-up study. Similarly, transmission electron microscopy points to the local order in the studied HfNbTaTiZr alloy.

\section{Acknowledgments}

The financial support by the Czech Science Foundation under project $17-17016 \mathrm{~S}$ is appreciated. First principles calculations performed were supported by the Czech Republic Ministry of Education, Youth and Sports from the Large Infrastructures for Research, Experimental Development and Innovations project "IT4Innovations National Supercomputing Center - LM2015070".

\section{References}

[1] D.B. Miracle, O.N. Senkov, Acta Mater. 122, 448 (2017).

[2] J.W. Yeh, S.K. Chen, S.J. Lin, J.Y. Gan, T.S. Chin, T.T. Shun, C.H. Tsau, S.Y. Chang, Adv. Eng. Mater. 6, 299 (2004).

[3] O.N. Senkov, J.M. Scott, S.V. Senkova, D.B. Miracle, C.F. Woodward, J. Alloys Comp. 509, 6043 (2011).

[4] O.N. Senkov, J.M. Scott, S.V. Senkova, F. Meisenkothen, D.B. Miracle, C.F. Woodward, J. Mater. Sci. 47, 4062 (2012).
[5] J. Zýka, J. Málek, Z. Pala, I. Andršová, J. Veselý, in: METAL 2015: 24th Int. Conf. on Metallurgy and Materials, TANGER Ltd, 2015, p. 1687.

[6] K. Ishikawa, Y. Tobita, Prog. Theor. Phys. 122, 1111 (2009)

[7] M.J. Puska, R.M. Nieminen, Rev. Mod. Phys. 66 , 841 (1994).

[8] K. Sato, H. Uchiyama, I. Kanazawa, E. Hamada, T. Suzuki, T. Takeuchi, U. Mizutani, J. Alloys Comp. 342, 306 (2002).

[9] O. Melikhova, J. Čížek, J. Kuriplach, I. Procházka, F. Lukáč, M. Cieslar, A. Anwand, G. Brauer, J. Phys. Conf. Ser. 265, 012016 (2011).

[10] A.D. Pogrebnjak, J. Nanomater. 2013, 780125 (2013).

[11] G. Kresse, J. Furthmüller, Phys. Rev. B 54, 11169 (1996).

[12] G. Kresse, D. Joubert, Phys. Rev. B 59, 1758 (1999).

[13] J.P. Perdew, A. Ruzsinszky, G.I. Csonka, O.A. Vydrov, G.E. Scuseria, L.A. Constantin, X. Zhou, K. Burke, Phys. Rev. Lett. 100, 136406 (2008).

[14] R.F.W. Bader, Atoms in Molecules: A Quantum Theory, Oxford University Press, 1991.

[15] W. Tang, E. Sanville, G. Henkelman, J. Phys. Condens. Matter 21, 084204 (2009).

[16] N. Metropolis, A.W. Rosenbluth, M.N. Rosenbluth, A.H. Teller, E. Teller, J. Chem. Phys. 21, 1087 (1953).

[17] J. Zýka, J. Málek, J. Veselý, F. Lukáč, J. Č́ížek, J. Kuriplach, O. Melikhova, Entropy 21, 114 (2019).

[18] J. Kuriplach, B. Barbiellini, Phys. Rev. B 89, 155111 (2014).

[19] J. Kuriplach, B. Barbiellini, AIP Conf. Proc. 1970 040006 (2018).

[20] P. Blaha, K. Schwarz, G.K.H. Madsen, D. Kvasnicka, J. Luitz, R. Laskowski, F. Tran, L.D. Marks, "WIEN2k, An Augmented Plane Wave plus Local Orbitals Program for Calculating Crystal Properties", Techn. Universität Wien, Austria 2001.

[21] B. Barbiellini, J. Kuriplach, Phys. Rev. Lett. 114, 147401 (2015).

[22] A.C.L. Jones, H.J. Rutbeck-Goldman, T.H. Hisakado, A.M. Piñeiro, H.W.K. Tom, A.P. Mills, B. Barbiellini, J. Kuriplach, Phys. Rev. Lett. 117, 216402 (2016).

[23] F. Lukáč, M. Dudr, J. Č́žek, P. Harcuba, T. Vlasák, M. Janeček, J. Kuriplach, J. Moon, H.S. Kim, J. Zýka, J. Málek, Acta Phys. Pol. A 134, 891 (2018).

[24] Y.Q. Xie, Acta Metall. Mater. 42, 3705 (1994).

[25] Y.Q. Xie, K. Peng, X.X. Yang, J. Cent. South Univ. Technol. 8, 83 (2001).

[26] S.Y. Chen, Y. Tong, K.-K. Tseng, J.-W. Yeh, J.D. Poplawsky, J.G. Wen, M.C. Gao, G. Kim, W. Chen, Y. Ren, R. Feng, W.D. Li, P.K. Liaw, Scr. Mater. 158, 50 (2019)

[27] M.J. Puska, R.M. Nieminen, J. Phys. F Met. Phys. 13, 333 (1983) 